\title{
Coil characteristic analysis and efficiency optimisation of wireless power transmission system
}

\section{Guanyu Chen}

Faculty of Engineering,

University of Nottingham,

Nottingham, UK

Email: chenguanyu931116@163.com

\begin{abstract}
How to charge more conveniently is a challenging problem, which affects people's daily life from two aspects of distance and portability. Considering the coil characteristics and coil size, the transmission system must adapt to these variables. Planar and layer wound coils are two widely used transmission coils. However, there is no further analysis on the layer wound coil in the previous publications. Compared with the planar structure, the layer wound structure has many advantages, such as longer transmission distance, faster energy accumulation speed. Therefore, there are more energy requirements in the scenarios of high-power electric locomotive and electric vehicle charging and household appliances. In addition, a new reference variable (coil turn spacing) is added in this paper, which is used to further optimise the system. Therefore, it is of great significance for the research of radio power transmission system.
\end{abstract}

Keywords: wireless power transmission; layer wound structure.

Reference to this paper should be made as follows: Chen, G. (2021) 'Coil characteristic analysis and efficiency optimisation of wireless power transmission system', Int. J. Cybernetics and Cyber-Physical Systems, Vol. 1, No. 1, pp.105-118.

Biographical notes: Guanyu Chen graduated with a bachelor's degree in electrical engineering from the University of the West of England in Bristol, in 2019 , and received a master's degree in electrical and electronics from the University of Nottingham, UK in 2020.

\section{Introduction}

Wireless power transmission technology is one of the most active research hotspots in recent years. Mechanical abrasion and burn-in are inherent defects of traditional contact transmission. Wireless power transmission has many advantages compared to traditional ones, such as convenient power supply, high reliability, and strong environmental adaptability (Kurs et al., 2007). According to different transmission principles, wireless 
power transmission can be divided into electric field coupling and electromagnetic coupling. Compared with the far-field transmission, the near-field transmission power is higher and has less impact on the environment (Garnica et al., 2013).

Magnetic coupling technology is introduced into the magnetic field power transmission, which extended the transmission distance and improved transmission efficiency (Mutashar et al., 2014). The effective coupling and high-quality factor of the transmission coil and the receiving coil are essential to the medium distance transmission of magnetically coupled radio energy (Khaleel et al., 2013). Because the system needs to work at a higher frequency (hundreds of $\mathrm{kHz}$ to tens of $\mathrm{MHz}$ ), the high-quality factor is unfavourable to the stability of the transmission system and the coil design (Soma et al., 1987). The design of effective coupling coil is the key to improve the transmission efficiency of the system. This paper shown that the transmission efficiency is affected by three factors: mutual inductance coefficient M, coil size and conductor radius. Improving the mutual inductance of coil is the key to study the transmission power and efficiency of the system (Yin and Ghovanloo, 2009). At present, the research of wireless power transmission does not consider the influence of coil position on system transmission (Ahn and Hong, 2014). In this paper, the transmission efficiency of the coil under horizontal displacement and the electromagnetic coupling characteristics of the layer wound coil is studied by simulation. The type of coil and the proportion of transceiver coil are analysed in depth. Case studies have made a lot of meaningful explorations on radio power transmission technology.

\section{A general review on wireless power transfer}

The most common near-field transmission system is magnetic field coupling, which can use magnetic energy to transmit electric energy. Liu et al. (2016) points out that the distance $(r)$ between transmitting coil and receiving coil should be less than one wavelength $(\chi)$ for near-field system. Besides, the maximum size of transmitting coil should be less than $\chi / 2$. On the other hand, for most near-field WPT methods, operating frequencies in the range of $100 \mathrm{kHz}$ to $50 \mathrm{MHz}$ are used. Liu et al. (2016) point out that the WPT system consists of two coils, called "primary coil" and "secondary coil". Therefore, transmission can be regarded as a near-field process. According to the electromagnetic theory, if the length of the receiving coil is smaller than that of the transmitting coil, it will be transmitted through the magnetic field within a certain distance, thus forming an energy transfer zone (Covic and Boys, 2013).

This kind of near-field transmission for inductive coupling has a long history in WPT. In early 2006, Beart was committed to fast wireless car charging. He described a near-field transmission in which the frequency varies in the range of $20-40 \mathrm{kHz}$, with about $10 \mathrm{~cm}$. The source driving the variable magnetic field is connected to the primary coil, which will sense a voltage on the receiver secondary coil and transfer the power transmission to the load accordingly (Beart et al., 2006). Due to the limitation of distance, Hui (2013) clearly solved this problem in his paper. He has done a lot of original work on radio power transmission. According to the flow direction of magnetic flux relative to charging surface, inductive coupling can be divided into two categories: horizontal approach and vertical approach. 
In the near-field transmission, Liu et al. (2016) discussed the eddy current phenomenon in induction charging, which may cause spark discharge and other hazards. Liu suggested to introduce a very thin electromagnetic shield under the charging plate and above the receiving coil to solve this problem. Also, in Chinga's paper, he designed an efficient wireless power transmission system using electromagnetic coupling to eliminate external metal contact of electronic devices (Chinga and Tseng, 2009). In addition, Shinohara (2010) mentioned in his paper that the transmission effect of layer wound coil is higher than that of planar coil. In the following sections, case studies will mainly study the characteristics and efficiency of the layer wound coil.

In 2006, Landis proposed the far-field power transmission technology, which is suitable for electromagnetic wave propagation in long distance (km range) with $R>2 L$ (Landis, 2006). In addition, he suggested using a microwave with a frequency of $2.45 \mathrm{GHz}$ as the emission source. In his study, it was shown that the size of the transmitter antenna is about $1 \mathrm{~km}$, while the rectifier and other equipment of the receiver need to be $10 \mathrm{~km}$. But this is not possible. According to the Shinohara report, it pointed out that reducing the wavelength can minimise the antenna size, but the disadvantage is to absorb the wave into the air (Shinohara, 2010).

Far field transmission has some advantages. Percy pointed out that the most important thing is that, compared with the near field, it has a longer transmission distance and is more efficient. In addition, Percy recommended using a power transmitter system in the rectifier at the transmitter to transmit rectified $10 \mathrm{~kW}$ power at $80 \%$ efficiency to power electric vehicles (Percy et al., 2014).

However, the disadvantages are obvious in practice. Erol-Kantarci and Mouftah (2014) discussed that the commercialisation of these systems depends on the design cost and the infrastructure, whereas the electromagnetic compatibility of these systems needs to be considered during the design. Besides, they are less robust and slower than near field. Due to the limitations, far-field transmission is usually used in some specific fields, such as space-based solar energy. In conclusion, near-field transmission will be used for further study in the following parts of this paper.

\section{Analysis of influence factors on the mutual inductance of coil}

The physical phenomenon that the coils are connected through their magnetic fields is called magnetic coupling. Mutual inductance coefficient $M$ is defined as:

$$
M=M_{12}=M_{21}=\frac{\psi_{12}}{I_{2}}=\frac{\psi_{21}}{I_{1}}
$$

When the two coils are parallel, the maximum mutual inductance coefficient can be obtained when the magnetic flux of the transmission channel is maximum. The equivalent circuit diagram of the system is shown in Figure 1. Currents flowing through the coils at the transmitter and receiver ends are $I_{1}$ and $I_{2}$, respectively. The direction is shown in Figure 1. The transmitter self-impedance is:

$$
Z_{1}=R_{1}+j X=R_{1}+j\left(\omega L_{1}-\frac{1}{\omega C_{1}}\right)
$$


The impedance of the receiver is:

$$
Z_{2}=R_{2}+j X_{2}=R_{2}+j\left(\omega L_{2}-\frac{1}{\omega C_{2}}\right)
$$

Therefore, the input and output power of the system can be derived.

$$
\begin{aligned}
& P_{\text {in }}=\mathrm{U}_{\mathrm{s}} \operatorname{Re}\left(I_{2}\right)=U_{s} \operatorname{Re}\left(\frac{U_{s} Z_{2}}{Z_{1} Z_{2}+(\omega M)^{2}}\right) \\
& P_{\text {out }}=\left(I_{2}\right)^{2} R_{L}=\left(\frac{U_{s} j \omega M}{Z_{1} Z_{2}+(\omega M)^{2}}\right)^{2} R_{L}
\end{aligned}
$$

Therefore, the efficiency of radio energy transmission system can be expressed as follows:

$$
\eta=\frac{P_{o u t}}{P_{\text {in }}}=\frac{(\omega M)^{2} R_{L}}{R_{1} X_{2}^{2}+R_{1}\left(R_{2}+R_{L}\right)^{2}+(\omega M)^{2}\left(R_{2}+R_{L}\right)}
$$

According to equations (4) and (5), it can be concluded that the mutual inductance coefficient $M$ is one of the most important factors affecting transmission power and efficiency.

Figure 1 Circuit model of inductive coupling

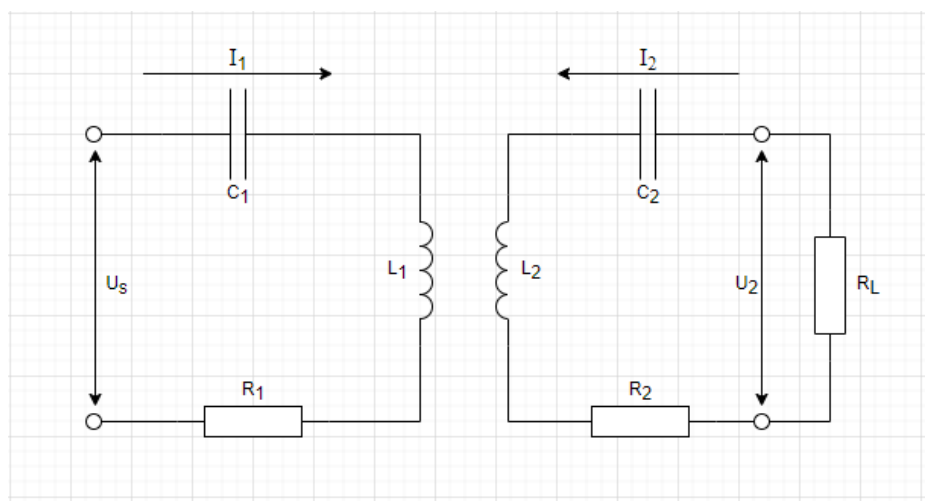

\section{Case studies}

Case 1: The size design of transmitting coil and receiving coil has a direct impact on transmission efficiency. Reasonable configuration of coil size can improve transmission level and efficiency.

When the distance between two coils is fixed at 2, 4, 6, 8 and 10 metres, the number of turns $N 1=N 2=15$. The curve of transmission efficiency versus radius $r$ can be obtained, as shown in Figure 2. 
Figure 2 The effect of different size coils on energy transfer

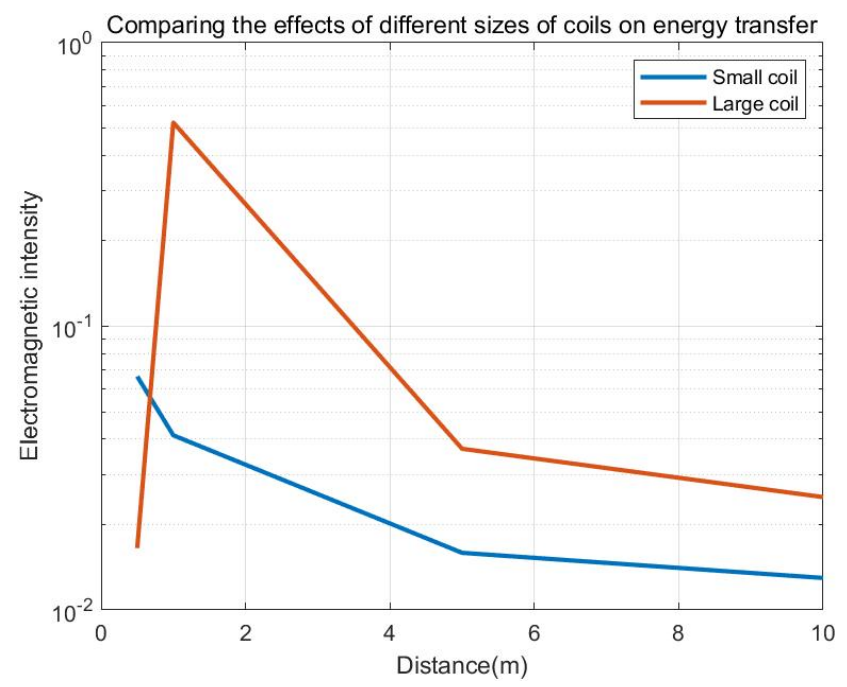

It can be seen from Figure 2 that, when the distance increases, the transmission efficiency of the small coil decreases with the increase of the coil radius $\mathrm{R}$. The transmission efficiency of the large coil increases first and then decreases with the increase of the coil radius, and there is an obvious pole value. Although different sizes of coils have different trends when the distance increases, the transmission efficiency decreases with the increase of spacing D. The advantage of small coil is short distance transmission, while large coil is more efficient in medium distance transmission.

The coil radius of $10 \mathrm{~cm}$ is set as the small coil, and the coil radius $30 \mathrm{~cm}$ is the large coil. Figure 2 shows that the attenuation rate of coil radius is different with different coil radius. The smaller radius and the better the near distance transmission effect, but the faster the attenuation speed. The coil radius is large, although the coupling effect is poor, the attenuation speed of the system is slow. The curve tends to be flat.

Therefore, small size coil is more effective for short distance transmission, and large size coil should be designed for long-distance transmission, but too small or too large coil radius is not conducive to the improvement of transmission efficiency. If the radius is too small, the attenuation of $\mathrm{M}$ is fast, which is not conducive to power transmission. However, if the radius is too large, the $m$ value is always low, which is not conducive to transmission. Therefore, when designing the coil size, the transmission distance should be fully considered, and the coil radius should match the distance.

Case 2: In this paper, the concept software is used to model and simulate the complex space magnetic field, and the magnetic field generated by different types of coils is analysed and calculated.

The simulation model is established according to the experimental parameters. The frequency is $10 \mathrm{MHz}$, and the radius of the transmitting coil is $2 \mathrm{~cm}$. Under the condition that the type and size of the transmitting coil and the receiving coil are unchanged, the coil distance and the horizontal lateral displacement of the receiving coil are simulated respectively. The results are shown in Figure 3 and Figure 4. The larger the magnetic flux density in the red region, the higher the coupling intensity; the smaller the magnetic flux density in the lighter part, the lower the coupling strength. 
Figure 3 Energy transfer at different distances

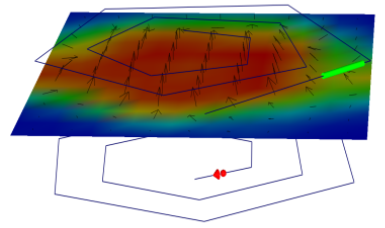

(a) Distance $=1$
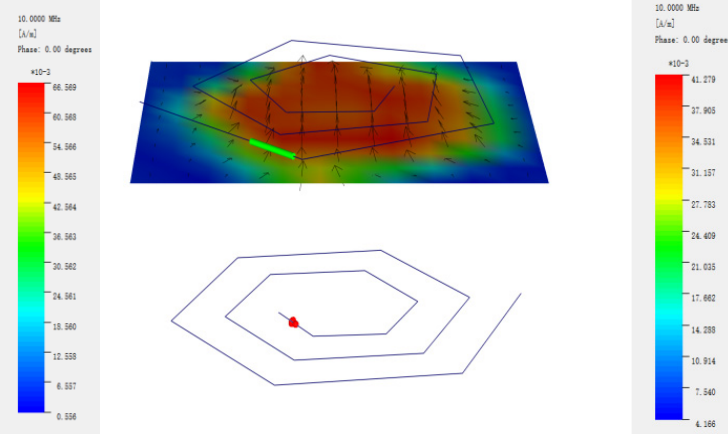

(b) Distance $=5$

Figure 4 Energy transfer at different traverse distances

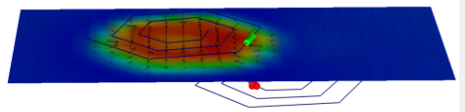

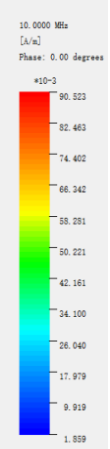

(a) Move one metre horizontally

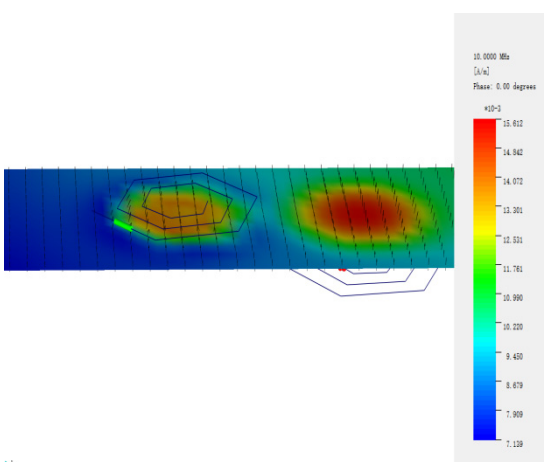

(b) Move two metres horizontally

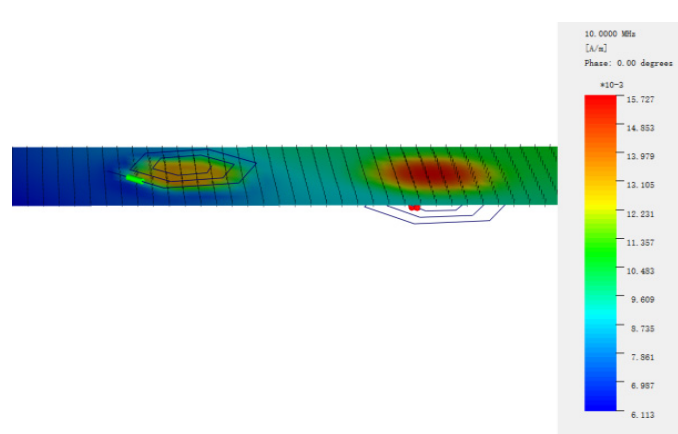

(c) Move three metres horizontally

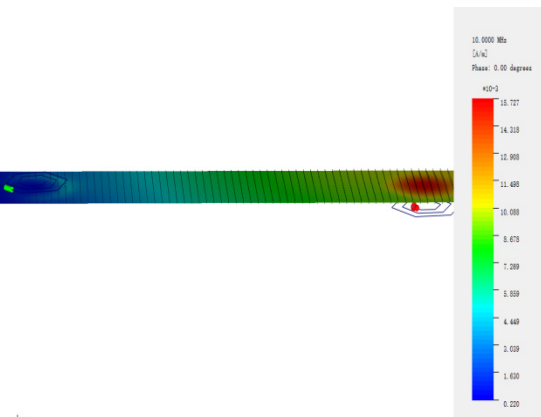

(d) Move four metres horizontally 
It can be seen from Figure 3 that the farther the distance between coils, the weaker the magnetic coupling strength. It is consistent with the theory of coil size and transmission distance.

From Figure 4, simultaneous interpreting of the location of the receiving coil also has a significant effect on the coupling strength of the magnetic field at the same transmission distance. When the receiving coil moves horizontally, the coupling degree of the system tends to weaken. When the receiving coil and the transmitting coil move horizontally for 4 metres, the magnetic field coupling between the receiving end and the transmitting end is weak. It is proved that the theoretical analysis based on the vertical parallel placement of coils is basically reasonable.

Figure 5 Compare the energy transfer under different traverse distances $(\mathrm{cm})$

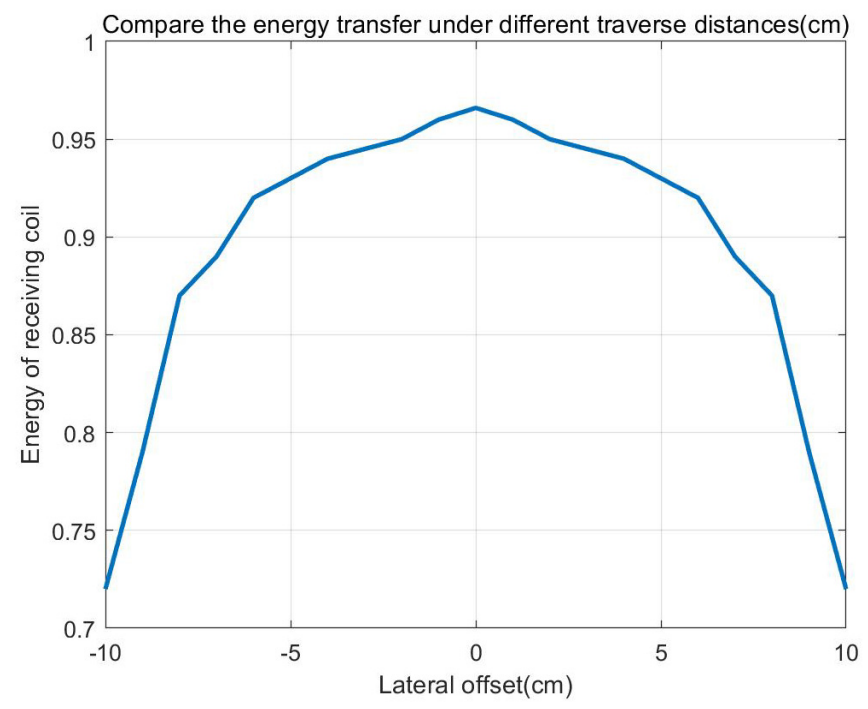

According to Figure 5, when the lateral offset is 0 , the energy transfer value is the maximum. With the lateral offset of the receiving coil, the energy transmission value tends to decay, and the larger the offset, the faster the attenuation speed of the transmission system. Because of the strongest symmetry of the circular coil, the lateral offset has no directionality and its system stability is the best. When the position of the receiving coil is shifted, the system has low attenuation rate and good charging stability.

As shown in the Figure 6, when the distance is between -10 metres and 10 metres, the stability of the system is not as good as before. In other words, when the lateral distance is large, the transmission efficiency of the system will decline rapidly.

Case 3: Under the same transmission distance, different sizes of receiving coils are simulated (Figure 7). The ratio coefficient of transmitting coil radius $R 1$ to receiving coil $R 2$ is $E=R 2 / R 1$. It can be seen from Figure 7(a) and (b) that when the radius of transmitting coil is larger than that of receiving coil $(e<1)$, the coupling degree between coils is higher. With the increase of the receiving coil, the coupling effect decreases obviously. It can be observed from Figure 7(d) that when $e=1.5$, the coupling degree is weak and there is no magnetic field coupling. 
Figure 6 Compare the energy transfer under different traverse distances (m)

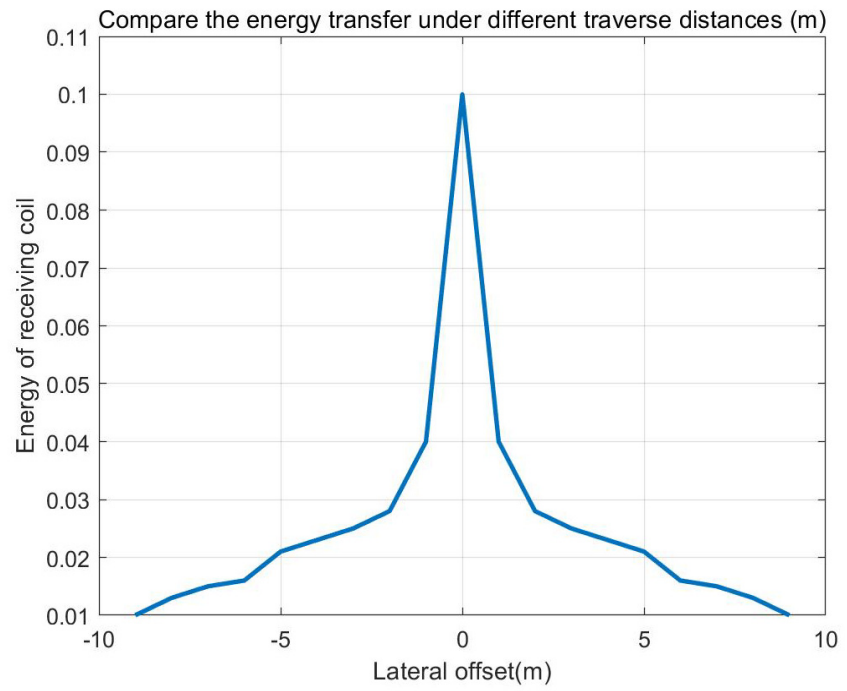

Figure 7 Compare the energy transfer under different traverse distances
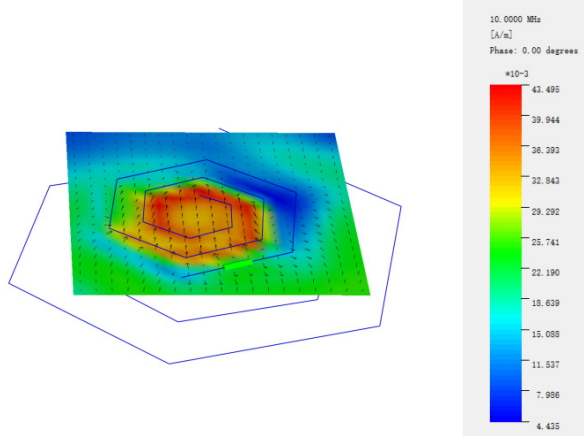

(a) $E=0.5$

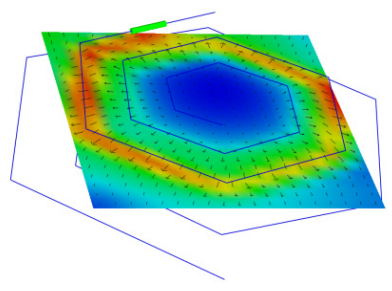

(b) $E=0.7$
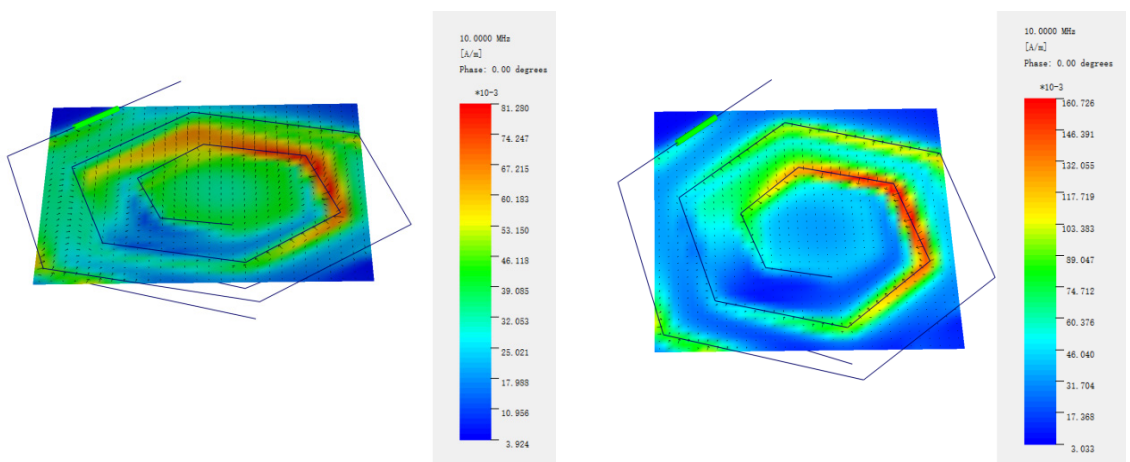

(c) $E=1.3$

(d) $E=1.5$ 
Therefore, in the design of coil size, the requirements of transmission distance and the selection of coil radius should be fully considered. The transmitting coil is larger than the receiving coil $(e<1)$, which can effectively improve the transmission efficiency.

Figure 8 Comparing the effects of different Scale factor of coils on energy transfer

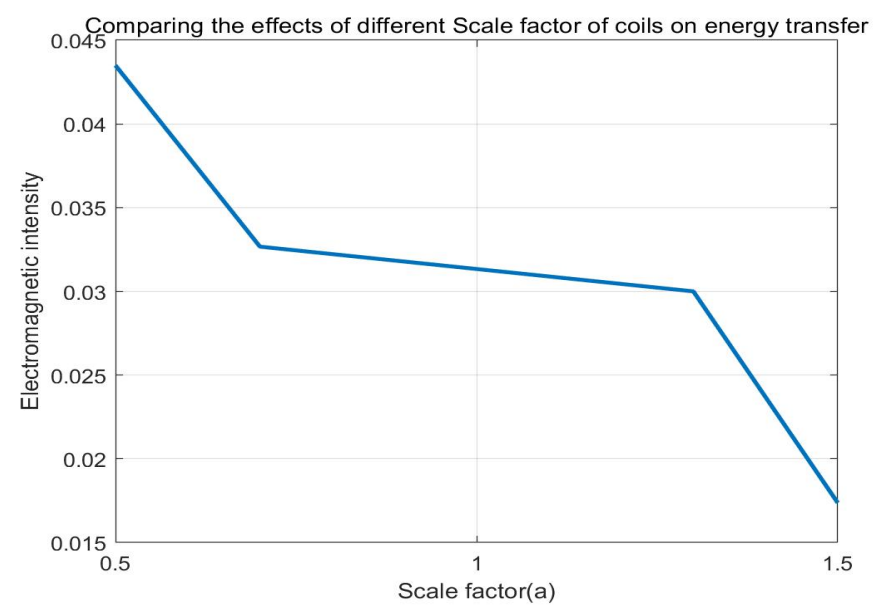

It can be seen from Figure 7 and Figure 8 that when the transmitting coil is larger than the receiving coil $(a<1)$, the coupling effect between the coils is better and the transmission efficiency is higher. From Figure 8, we can intuitively get the importance of the scale factor of the coil.

In these three cases, the principle and composition of wireless power transmission system are analysed in detail. Based on the radio power transmission of double coil structure, the transmission efficiency of the system is analysed in detail from three aspects: coil function, wire radius and coil size. The advantages and disadvantages of planar structure are summarised. The data of the model are sorted out with MATLAB, which provides a reference for subsequent research.

Case 4: The magnetic field distribution of two kinds of coils with different structures is shown in Figure 9. It can be seen from Figure 9 that the electromagnetic coupling effect of layer wound coil is better than that of planar structure coil, and the adoption of layer wound structure coil has certain significance for improving the coupling degree of coil. This is due to the tunnelling effect in the electromagnetic field, and the coupling transmission process is the same as the quantum tunnelling effect.

According to Figure 10, it can be seen that when the two types of coils are horizontally offset, the transmission efficiency of the layer wound coil is higher. Through the changing trend, there are obvious differences between the two kinds of coils, which also shows that the layer wound structure is more stable in the offset. And from the simulation results, we can see that the layer wound coil can achieve higher transmission efficiency under the condition of constant parameters. 
Figure 9 Magnetic field distribution of two different coils
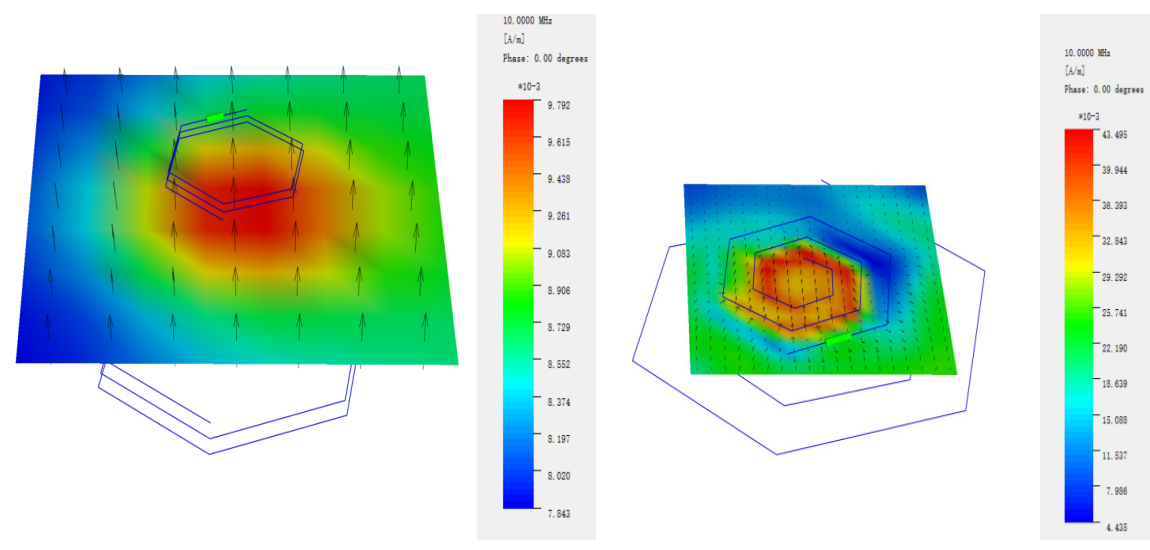

Figure 10 Transfer efficiency of two coils varies with horizontal displacement

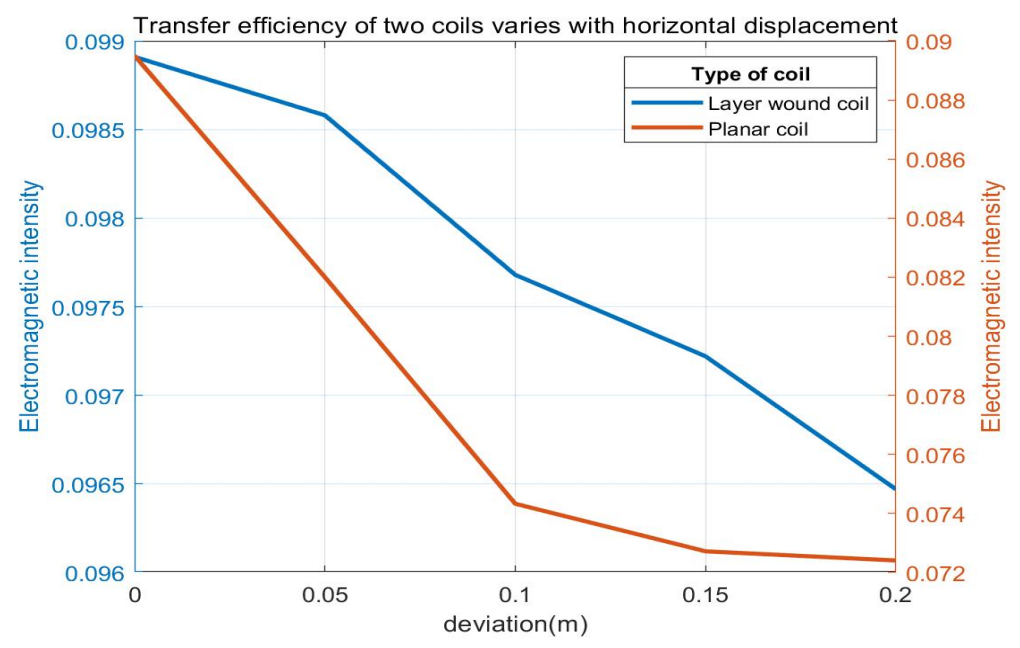

Case 5: The coil turn spacing is not mentioned in the Journal of Power Transmission. However, the size of coil turn spacing can improve the transmission capacity of the system to a certain extent. If the coil turn spacing is too small, the system will not work properly. Generally, the tightness of the coil indicates the distance between each turn of the coil. In the design of coil, in order to meet the normal operation of the system, appropriate reservation of turn distance can improve the transmission efficiency of coil, thus saving cost and improving transmission efficiency. However, when the coil turn spacing is too large, the energy will spread too fast in the transmission process, which will also have a negative impact on the transmission efficiency of the system. In order to get the best coil turn distance through visual analysis, the transmitting coil and receiving coil are 15 turns, and the vertical distance is $0.5 \mathrm{~m}$, and the coil turn spacing is constantly changed to observe its influence. 
As shown in Figure 11, under the simulation of CONCEPT software, the turn spacing of the coil starts from $0.8 \mathrm{~mm}$, and then takes $0.5 \mathrm{~mm}$ as the step size, with the value between $0.8 \mathrm{~mm}$ and $1 \mathrm{~mm}$. With the help of data collection function of MATLAB, the corresponding energy value under each simulation parameter is drawn in a coordinate.

Figure 11 Comparing the effect of different coil spacing per turn on the layer winding structure

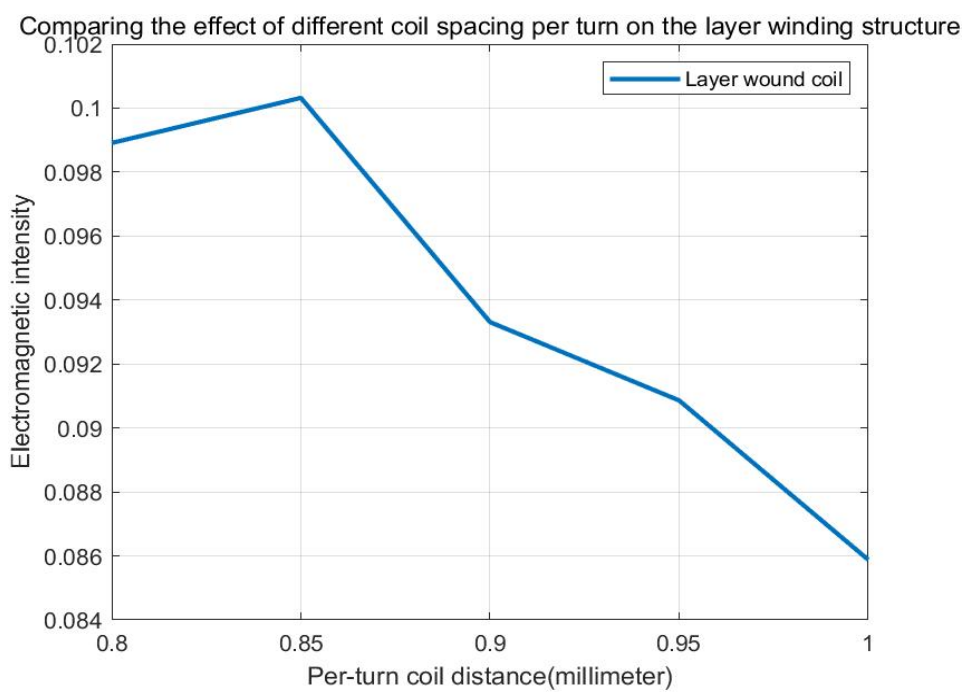

In the process of increasing the coil turn spacing, the transmission capacity of the system first increases and then decreases. Therefore, when the turn spacing of the coil is between $0.8 \mathrm{~mm}$ and $0.85 \mathrm{~mm}$, the cost can be saved, and the copper loss can be reduced. The simulation results show that when the coil turn spacing exceeds $0.85 \mathrm{~mm}$, the transmission capacity of the coil decreases. Therefore, in the production and design of transmission coil, it is necessary to test the optimal coil turn spacing to improve the transmission efficiency.

Case 6: After the above analysis and modelling, the transmission efficiencies of large coil, small coil and layer wound coil are compared. In addition, a new reference variable is introduced, that is, the turn spacing of the coil. But the load variable also needs to be discussed. Wireless power transmission can be divided into power supply, transmitting coil, receiving coil and load. These four parts restrict each other and affect the transmission characteristics of the whole system. Previous experimental studies mainly focus on coil characteristics and coil design, but the research on load and system adaptability is relatively poor. For wireless energy transmission system, the adaptability of different loads to the system is different. After the above analysis and modelling, we have a more in-depth understanding of the composition and impact of the radio power transmission system. In order to explore the impact of load on transmission performance, Conric was used to build the system model. Conric can realise the dynamic modelling of the system. The simulation model is shown in Figure 12. 
Figure 12 Wireless power transmission circuit diagram

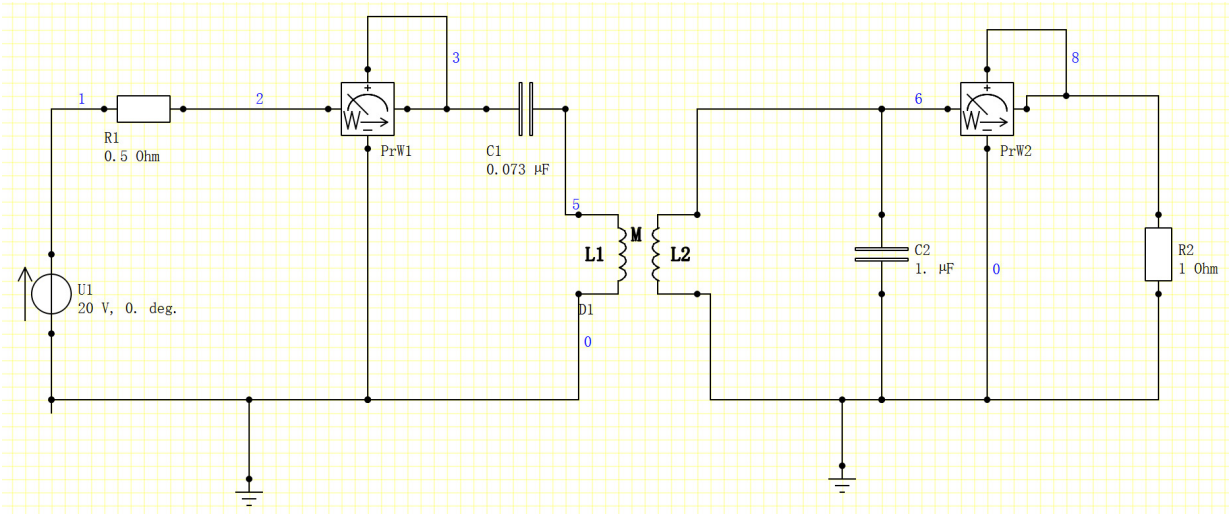

Therefore, it is necessary to find the best load point. As shown in Figure 13, the system achieves the highest transmission efficiency at the red marking point.

Figure 13 Wireless power transmission efficiency under different loads

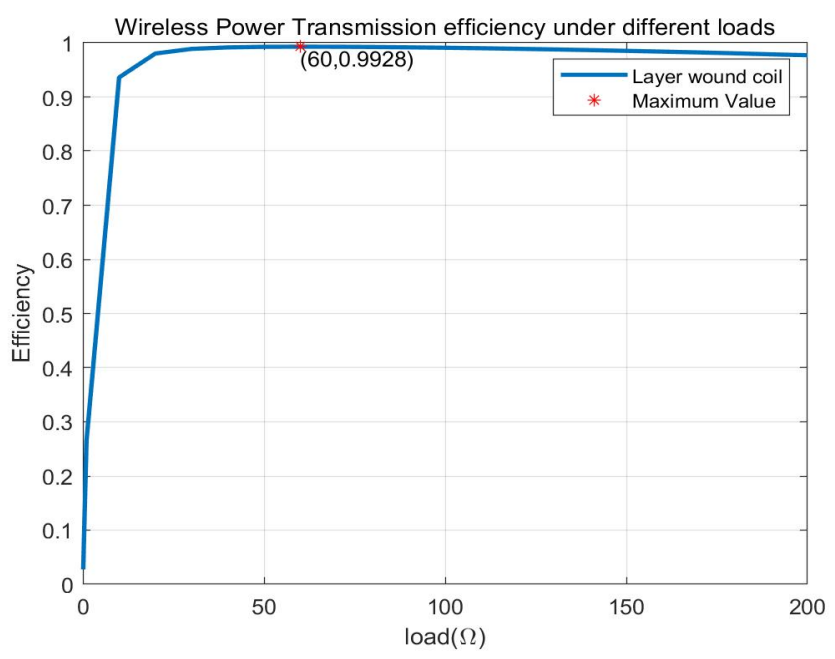

As shown in Figure 13, the load efficiency first increases and then decreases with the increase of load resistance, and there is a load value to maximise the output efficiency of the system. It is called the highest ideal efficiency load point. In this simulation, the maximum efficiency load point is about $60 \mathrm{ohm}$ and the efficiency is about $98 \%$. When the load is less than this point, the reduction of load will lead to the sharp decline of system efficiency. When the load is $1 \mathrm{ohm}$, the transmission efficiency of the system is only $70 \%$. When the load is greater than $60 \mathrm{ohm}$, the transmission of the system will show a slow downward trend. When the load is $190 \mathrm{ohm}$, the transmission efficiency is about 90\%. 


\section{Conclusions}

The need for radio energy charging has attracted extensive attention. Planar coil is the most traditional transmission structure, but the transmission efficiency and space design are not well reflected. Systems based on layer-wound structural coils can transmit effective energy. Using near-field transmission as the application background, the layerwound coil is modelled and analysed to improve the transmission efficiency which puts emphasis on the factors of transmission distance and horizontal displacement. The system is optimised by reasonable coil design and increasing coil turn spacing. The work of this paper and the conclusions drawn are as follows:

1 In these six case studies, the coil characteristics and system coupling mechanism are analysed. Firstly, based on the analysis of coil energy transmission, the expression of coil characteristics on efficiency is derived. In concept simulation, the influence of coil size, transmission direction and coil structure on the system is concluded. Secondly, the comparison and simulation of layer wound coil and planar structure are carried out. In order to comprehensively consider the performance of the layer wound coil, in addition to the basic characteristics of the coil, two new reference variables, the turn spacing of the coil and the load selection of the receiving coil, are introduced in the optimisation design. Finally, the optimal coil structure suitable for the system is determined.

2 The expression for the mutual inductance coefficient $M$ of two coils is derived, and the coil distance, size and offset are studied. When the receiving coil offset is small, it has good offset characteristics; when the receiving coil offset is large, the transmission efficiency is reduced

3 In the system design, it is necessary to make two coils vertical and parallel to increase the number of coil turns, and the receiving coil is smaller than the transmitting coil, which is beneficial to improve the transmission of the system. The use of layer wound coil can improve the efficiency and effective transmission range of wireless transmission.

4 The coil size should be designed according to the requirements of transmission distance. For short distance transmission, the transmitting coil should be larger than the receiving coil radius, which is conducive to improving the coupling effect. The ratio coefficient of receiving coil and transmitting coil is $1<e<1.5$.

\section{References}

Ahn, D. and Hong, S. (2014) 'Wireless power transmission with self-regulated output voltage for biomedical implant', IEEE Transactions on Industrial Electronics, Vol. 61, No. 5, pp.2225-2235.

Beart, P., Cheng, L. and Hay, J. (2016) Inductive energy transfer system having a horizontal magnetic field, U.K. Patent GB2399225.

Chinga, R.A. and Tseng, R. (2009) 'Design and test of a high-power high-efficiency loosely coupled planar wireless power transfer system', IEEE Trans. Industrial Electronics, Vol. 56, No. 5, pp.1801-1812.

Covic, G.A. and Boys, J.T. (2013) 'Inductive power transfer', Proceedings of the IEEE, Vol. 101, No. 6, pp.1276-1289. 
Erol-Kantarci, M. and Mouftah, H.T. (2014) 'Radio-frequency-based wireless energy transfer in LTE-A heterogeneous networks', Proceedings of IEEE Symposium on Computers and Communication (ISCC), Funchal, Portugal.

Garnica, J., Chinga, R.A. and Lin, J. (2013) 'Wireless power transmission: from far field to near field', Proceedings of the IEEE, Vol. 101, No. 6, pp.1321-1331.

Hui, S. (2013) 'Planar wireless charging technology for portable electronic products and Qi', Proceedings of the IEEE, Vol. 101, No. 6, pp.1290-1301.

Khaleel, R.H., Al-Rizzo, M.H. and Abbosh, I.A. (2013) 'Design, fabrication, and testing of flexible antennas', Advancement in Microstrip Antennas with Recent Applications, pp.363-383.

Kurs, A., Karalis, A., Moffatt, R., Joannopoulos, J.D., Fisher, P. and Soljacic, M. (2007) 'Wireless power transfer via strongly coupled magnetic resonances', Science, Vol. 317, pp.83-86.

Landis, G. (2006) 'Reevaluating satellite solar power system for Earth', IEEE 4th World Conference on Photovoltaic Energy Conversion, Waikoloa, Hawaii.

Liu, C., Guo, Y. and Xiao, S. (2016) 'A review of implantable antennas for wireless biomedical devices', Forum for Electromagnetic Research Methods and Application Technologies (FERMAT), Vol. 14.

Mutashar, S., Hannan, M., Samad, S. and Hussain, A. (2014) 'Analysis and optimization of spiral circular inductive coupling link for bio-implanted applications on air and within human tissue', Sensors, Vol. 14, No. 7, pp.11522-11541.

Percy, U., Knight, C., Cooray, F. and Smart, K. (2014) 'Supplying the power requirements to a sensor network using radio frequency power transfer', Sensors, Vol. 12, No. 7, pp.8571-8585.

Shinohara, N. (2010) 'Beam efficiency of wireless power transmission via radio waves from short range to long range', Journal of the Korean Institute of Electromagnetic Engineering and Science, Vol. 10, No. 4, pp.224-230.

Soma, M., Galbraith, D.C. and White, R.L. (1987) 'Radio-frequency coils in implantable devices: misalignment analysis and design procedure', IEEE Transactions on Biomedical Engineering, Vol. BME-34, No. 4, pp.276-282.

Yin, Y. and Ghovanloo, M. (2009) 'A flexible clockless 32-ch simultaneous wireless neural recording system with adjustable resolution', IEEE International Solid-State Circuits Conference - Digest of Technical Papers, San Francisco, CA, USA, pp.432-434. 